\title{
Differential Equation Model on the Prevalence of Diabetic Neuropathy
}

\author{
P.Sankar $^{1^{*}}$, M.S. Paulraj ${ }^{2}$, J.Jayanthi ${ }^{3}$ and M.G. Ragunathan ${ }^{3}$ \\ ${ }^{1}$ Department of Mathematics, Guru Nanak College, Chennai-42, India \\ ${ }^{2}$ Department of Mathematics, A.M. Jain College, Chennai-114, India \\ ${ }^{3}$ Department of Advanced Zoology and Biotechnology, Guru Nanak College, Chennai-42, India \\ Corresponding Author: sankargnc1@gmail.com, Tel.: 9445269155
}

\section{Available online at: www.isroset.org}

Accepted 25/Aug/2018, Online 30/Aug/2018

\begin{abstract}
Diabetic neuropathy is the most common complication of diabetes mellitus, and can be related to Type1 as well as Type2 diabetes. This neurological impairment is not known or understood; hence the treatments are still empirical and not efficient as stated by the physicians. The purpose of this study is to discuss the population based Mathematical model of diabetic neuropathy patients. The study adopted in Ordinary Differential Equations which contains the percentage of diabetic patients with and without diabetic neuropathy. By appropriate definition of a parameter, the solution discussed the critical nature of percentage of diabetic patients with and without diabetic neuropathy is discussed for stability.
\end{abstract}

Keywords - Diabetes, Diabetic Neuropathy, Classification, Ordinary Differential Equations.

\section{INTRODUCTION}

The incidence of diabetic is increasing because people are living longer, getting fatter and leading inactive life style. Diabetes is caused by increased level of glucose in blood. When carbohydrate is taken by body it get converted into glucose which transmitted to blood cells through blood with the help of insulin. Insulin has an important role for the control of intermediately metabolism. In case of normal human being, insulin is released from cells in the pancreas which usually absorb the excess glucose present in the blood and convert it into energy or fuel. Insulin also stimulates liver to absorb and store any glucose left over [1].

Diabetes mellitus is a metabolic disorder characterized by the decreased ability or complete inability of the tissues to utilize carbohydrate. The disorder is due to deficiency or diminished effectiveness of the hormone insulin [1]

\section{TYPE 1 DIABETES}

Type1 diabetes or insulin dependent diabetes mellitus (IDDM) is an autoimmune disease that causes the destruction of the pancreatic cells producing the insulin, resulting in hyperglycemia. It accounts for $10 \%$ of cases of diabetes and generally appears before the age of 20 , most often around the age of 12. Since the pancreas is unable to produce insulin in a quantity sufficient to prevent hyperglycemia, the treatment of Type 1 diabetes requires the regular administration of insulin. It also requires an adapted diet and frequent selftesting of glycaemia by capillary sampling. Left untreated, the disease can result in a coma, or even death. At the onset of the disease, the patient often does not feel any symptoms. When glycaemia reaches very high values, the body tries to eliminate the excess glucose by increasing the frequency and abundance of urination, and by producing urine with high sugar content. Dehydration follows, which causes intense thirst. Appetite increases, but this generally does not prevent weight loss. Type 1 diabetes also causes fatigue and headaches, and may promote repeated infections.

\section{TYPE 2 DIABETES}

Type 2 diabetes or non-insulin dependent diabetes mellitus (NIDDM), is a chronic disease characterized by the resistance of the body's cells to the action of the insulin produced by the pancreas. The cells assimilate glucose poorly and it accumulates in the blood (hyperglycemia). As a result, the pancreas provides more and more insulin to lower glycaemia, without effect. It becomes progressively exhausted, resulting in a decrease in insulin production that must be compensated for through injections. Type 2 diabetes is connected with aging, obesity, a sedentary lifestyle, as well as genetic factors that are poorly understood. It is increasing in industrialized countries and generally appears around age 50 , but it is more and more common in younger people. It may remain asymptomatic for several years before revealing 
itself through a complication. Its treatment is based on reducing sugar and fats in the diet, increasing physical activity, and the administration of hypoglycemic drugs or insulin. In 1985 Type 1 and Type 2 names were omitted and only IDDM \& NIDDM were known as the types of Diabetes Mellitus [2].

The majority of people with diabetes mellitus are NIDDM (Non-Insulin Dependent Diabetes Mellitus). Sometimes it becomes difficult to distinguish between IDDM and NIDDM. IDDM is usually diagnosed before the age of 40 and is treated by insulin injection and diet but NIDDM is usually diagnosed after the age of 40 and may be treated by diet alone or by diet and tablets both [3].

\section{Diabetes Mellitus IN INDIA}

Diabetes mellitus is the disease of millions and if the projections are right, a quarter billion people across the globe will be diabetic by the year 2025 . The prevalence of diabetes mellitus for all age groups worldwide was estimated to be $2.8 \%$ in 2000 and an anticipated $4.4 \%$ in 2030. The total number of people in the world with diabetes is projected to rise from 171 million in 2000 to 366 million in 2030 [4]. India ranked number one as the country with the highest number of diabetes patients in 1995, at 31.7 million in 2000, with a projected 57.2 million in 2025 , and 79.4 million in2030, retaining its top position [5].

\section{DIABETIC NeUROPATHY}

Diabetic neuropathies are a family of nerve disorders caused by diabetes. People with diabetes can, over time, develop nerve damage throughout the body. Some people with nerve damage have no symptoms. Others may have symptoms such as pain, tingling, or numbness - loss of feeling - in the hands, arms, feet, and legs. Nerve problems can occur in every organ system, including the digestive tract, heart, and sex organs. About 60 to 70 percent of people with diabetes have some form of neuropathy. People with diabetes can develop nerve problems at any time, but risk rises with age and longer duration of diabetes. The highest rates of neuropathy are among people who have had diabetes for at least 25 years. Diabetic neuropathies also appear to be more common in people who have problems controlling their blood glucose, also called blood sugar, as well as those with high levels of blood fat and blood pressure and those who are overweight.

\section{MATERIALS AND METHODS}

The dataset used in the study has been collected from the Dr. Mohan diabetes specialities centre database. The database containing 1393 records and each record containing several information including demographic particulars of the patients, family history, personal habits and food consumption behaviour were collected. A family history is described as any member previously had been diagnosed as having diabetes by a physician. Height and weight of the patients were also recorded. BMI was calculated. SPSS version 24 (IBM Corporation) was used for all statistical analysis.

An ordinary differential equations and numerical approximations are used to monitor the size of the diabetic patients with and without diabetic neuropathy.

\section{MATHEMATiCal MODEL}

A Mathematical model of diabetic patients will be examined which contains the percentage of diabetic patients with and without diabetic neuropathy. Finally this model gives the size of the diabetic patients in both with and without diabetic neuropathy. In this study we use the Runge-Kutta method to solve this Mathematical model.

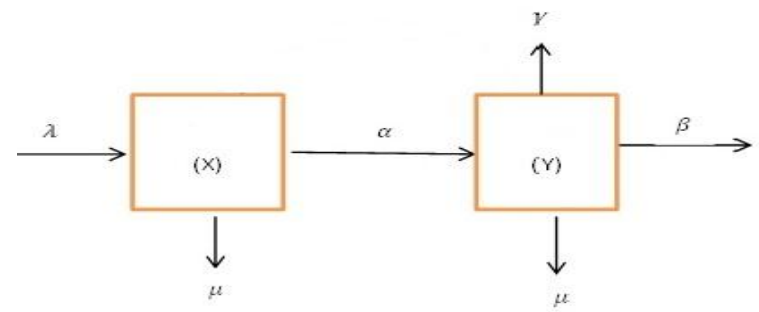

$\lambda$ : Incidence of diabetic mellitus (constant) $\{$ no. of cases diagnosed at a time $\mathrm{t}$ \}

$X=X(t):$ Percentage of patients without diabetic neuropathy at time $\mathrm{t}$.

$Y=Y(t)$ : Percentage of patients with diabetic neuropathy at time $\mathrm{t}$.

$\mu$ : Natural mortality rate percent.

$\alpha$ : Probability of diabetic person developing diabetic neuropathy.

$\beta$ : The rate percent at which diabetic neuropathy patients becomes severely disabled.

$\gamma$ : The mortality rate percent due to uncontrolled diabetic neuropathy.

Rate of change of diabetic patients with and without diabetic neuropathy is defined by Ordinary Differential Equations.

$$
\begin{gathered}
X^{\prime}(t)=\frac{d X}{d t}=\lambda-(\alpha+\mu) X \\
Y^{\prime}(t)=\frac{d Y}{d t}=\alpha X-(\mu+\beta+\gamma) Y
\end{gathered}
$$

$$
\text { Let } u=\alpha+\mu \text { and } v=\mu+\beta+\gamma
$$

Then equation (1) and (2) becomes

$$
X^{\prime}(t)=\frac{d X}{d t}=\lambda-u X
$$




$$
\begin{aligned}
& Y^{\prime}(t)=\alpha X-v Y \\
& \text { Let } \quad X^{\prime}=G(X, Y) \text { and } Y^{\prime}=H(X, Y) .
\end{aligned}
$$

We assume the initial conditions as

$$
X(0)=X_{0}, Y(0)=Y_{0}
$$

Now by Runge-Kutta method the solution of (3) and (4) is given by

$$
\begin{aligned}
& X_{n+1}=X_{n}+\frac{1}{6}\left(k_{1}+2 k_{2}+2 k_{3}+k_{4}\right) \\
& Y_{n+1}=Y_{n}+\frac{1}{6}\left(l_{1}+2 l_{2}+2 l_{3}+l_{4}\right)
\end{aligned}
$$

Where

$$
\begin{aligned}
& k_{1}=h G\left(X_{0}, Y_{0}\right) \quad l_{1}=h H\left(X_{0}, Y_{0}\right) \\
& k_{2}=h G\left(X_{0}+\frac{k_{1}}{2}, Y_{0}+\frac{l_{1}}{2}\right) \\
& l_{2}=h H\left(X_{0}+\frac{k_{1}}{2}, Y_{0}+\frac{l_{1}}{2}\right) \\
& k_{3}=h G\left(X_{0}+\frac{k_{2}}{2}, Y_{0}+\frac{l_{2}}{2}\right) \\
& l_{3}=h H\left(X_{0}+\frac{k_{2}}{2}, Y_{0}+\frac{l_{2}}{2}\right) \\
& k_{4}=h G\left(X_{0}+k_{3}, Y_{0}+l_{3}\right) \\
& l_{4}=h H\left(X_{0}+k_{3}, Y_{0}+l_{3}\right)
\end{aligned}
$$

\section{NUMERICAL SOLUTIONS}

This figure shows that the percentage diabetic patients in both with and without diabetic neuropathy for 105 years. Mat lab 9.4 is used to solve the equations (1) and (2). The behavior of graph reached the steady state after 65 years.

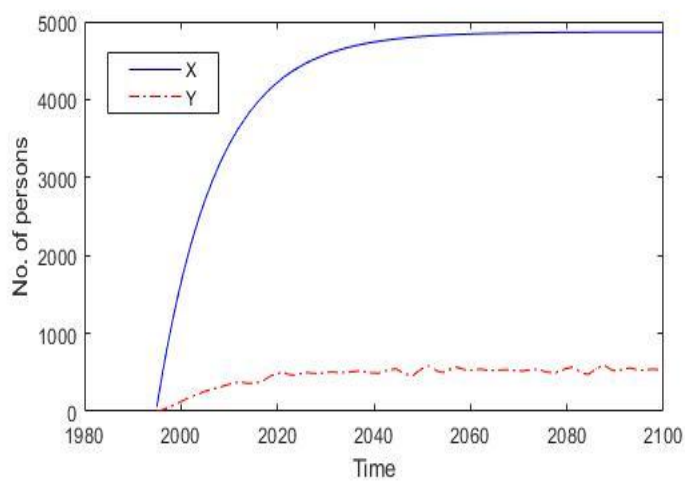

Fig.2
The detailed solution of Mathematical model is given below.

Total number of patients $(\lambda)=1393$

Number of patients without diabetic neuropathy

$$
X(t)=1017
$$

Number of patients with diabetic neuropathy

$$
Y(t)=376
$$

When $t=0$, Percentage of patients without diabetic neuropathy

$$
X_{0}(0)=\frac{1017}{1393}=73
$$

Percentage of patients with diabetic neuropathy

$$
Y_{0}(0)=\frac{376}{1393}=27
$$

Naturally mortality rate percent $(\mu)=0.02$

The rate percent at which diabetic neuropathy patients becomes severely disabled $(\beta)=\frac{6}{376}=1.6$

The mortality rate percent due to uncontrolled diabetic

$$
\begin{aligned}
& \text { neuropathy patients }(\gamma)=\frac{1}{376}=0.3 \\
& N_{0}=X_{0}+Y_{0}=100 \\
& v=\mu+\beta+\gamma=1.92 \\
& \alpha=\frac{v Y_{0}}{N_{0}}=0.52 \\
& u=\alpha+\mu=0.54
\end{aligned}
$$

\section{CONCLUSION}

In this study, a Mathematical model which contains the percentage of diabetic patients with and without diabetic neuropathy. It is concluded that stability is obtained in both with and without diabetic neuropathy patients.

\section{ACKNOWLEDGEMENT}

We gratefully acknowledge Dr. R. Rajaji, Assistant Professor, Department of Mathematics, Patrician College,

Chennai for helping in compilation of this article.

\section{REFERENCES}

[1] Sandhya, Deepak Kumar, and Prerna Pandit; An Ordinary Differential Equation Model ofdiabetic population in NewDelhi. Indian Journal of Mathematics and Mathematical Sciences Vol. 7, No. 1, (June 2011): 45-50R. 
[2] Sarah Wild, Gojka Roglic, Anders Green, Richard Sicree, and Hilary King, (2004), Global Prevalence of Diabetes, Estimates for the Year 2000 and Projections for 2030.Diabetes Care, 27, 10471053.

[3] A. Boutayeb, A.Chetouani, A. Achouyab, and E. H. Twizell, (2006), A Non-LinearPopulation Model of Diabetes Mellitus, J. Appl. Math. \& Computing, 21(1), 127-139.

[4] Jitender Nagpal, and Abhishek Bhartia, (2006), Quality of Diabetes Care in the Middle-and High-Income Group Populace, Diabetes Care, 27, 1047-1053.

[5] Deepak Kumar, Sandhya, Anuj Kumar, and Prerna Pandit, (2009), A ComputationalModel for Diabetes Mellitus, Optimization-Journal of Research and Management, 39-43.

\section{AUTHORS PROFILE}

P. Sankar, Assistant Professor in Mathematics, Guru Nanak College, Chennai has an in depth knowledge in Mathematics and a well experienced teacher with 21 years of experience. His field of specialization is Analytic Number Theory.

Dr. M.S. Paulraj, Associate Professor in Mathematics, A. M. Jain College, Chennai has an experience of 25 years in both teaching. One scholar awarded Ph.D and 19 students awarded M.Phil., degree under his guidance.

Dr. J. Jayanthi, Associate Professor in Zoology and Deputy Director, Gill Research Institute, Guru Nanak College, Chennai is a high profile Researcher, having completed 15 Ph.D., published 75 Research papers with Nationally and Internationally. Her Research experience dated back from two
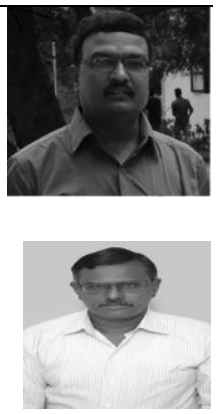
decades. Her field of specialization is crustacean biochemistry.

Dr. M.G. Ragunathan, Associate Professor in Zoology, Guru Nanak College, Chennai has a vast experience of 29 years in both teaching and Research. He has guided more than 25 Ph.D., and guiding 9 students currently. He has a publication list of more than 120 papers in reputed refereed journals of high impact value. $\mathrm{He}$ is a council member in the Indian Science Congress

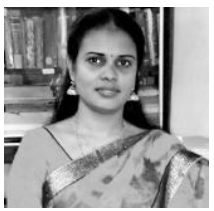
Association. 